\title{
GNSS BASED RELATIVE NAVIGATION FOR INTENTIONAL APPROXIMATION OF AIRCRAFT
}

\author{
Emmanuel SCHIELIN ${ }^{1}$, Thomas DAUTERMANN ${ }^{2}$ \\ ${ }^{1}$ SAGEM, Inertial Measurement Unit R\&D Department, 18/20 quai du Point du Jour, \\ 92659 Boulogne-Billancourt Cedex, France \\ ${ }^{2}$ German Aerospace Centre (DLR), Institute of Flight Guidance, Lilienthalplatz 7, \\ 38108 Braunschweig, Germany \\ E-mail: thomas.dautermann@dlr.de (corresponding author)
}

Received 03 April 2014; accepted 20 January 2015

Emmanuel SCHIELIN, $M S c$

Education: 2011 - Ecole Nationale de l'Aviation Civile (Toulouse, France), Master's Degree in aeronautical telecommunications.

Affiliations and functions: 2011-2013 - FDC (Paris, France): study engineer in the field of GNSS standardization and authentication; since October 2014 - system engineer at SAGEM (part of the SAFRAN group) in the Inertial Measurement Unit R\&D Department.

Research interests: satellite and inertial navigation.

Publications: coauthor of 1 book.

\section{Thomas DAUTERMANN, $\mathrm{Dr}$}

Education: Purdue University, 2004 MSc in Physics. Purdue University, 2008, PhD in Physics.

Affiliations and functions: since 2008 - German Aerospace Centre DLR, researcher and project manager.

Holds commercial pilots licenses from the United States and Europe.

Research interests: flight guidance and satellite navigation.

Publications: author of 8 papers; coauthor of 4 papers.

\begin{abstract}
In aviation, satellite navigation is generally only used to determine the absolute position of aircraft. We show that the signals can also be used for safe relative navigation provided that a data link exists between the two aircraft. The link can be used to form a double difference combination of code phase measurements and determine a three dimensional baseline vector. The baseline vector is protected by protection levels which determine the $3 \times 10^{-7}$ error bound of the baseline estimation. Thus, the distance vector can be used to perform safe approximation maneuvers in instrument weather conditions. We derive the protection level expression and test the baseline vector estimation using data from two real satellite navigation receivers on the ground. Moreover, we simulate an intercept mission using a Spirent GNSS7790 simulator and show that with the derived protection bounds an approximation up to $10 \mathrm{~m}$ is possible.
\end{abstract}

Keywords: GNSS, satellite navigation, aircraft, signals, simulation.

\section{Introduction}

Before the creation of the Global Positioning System (GPS), navigation in the sea, on the ground or in the air was approximate and not very precise. Indeed, at the beginning of navigation, humans used fixed stars, sun and moon to sail around the earth. The methods did not need an infrastructure nor any other equipment, apart form clocks; however, they were not accurate and robust (for example, a cloud could mask the stars). Since then, other techniques have been invented. Some are robust but need an infrastructure like the hyperbolic navigation system LORAN and some are only applicable locally like dead-reckoning. Others are world wide solutions, but are not precise over long distances. Inertial sensors for instance can measure the acceleration and rotation of an object in three dimensions, but drift over time and need to be reset again after a certain period. The creation of the American Navstar Global Positioning System (GPS, a constellation of satellites, which send signals to the user who can then compute his position 
from the visible satellites) created a revolution by providing users with accurate navigation information. The precision is at the order of meters, but can decrease to a few millimeters using augmentation systems like Satellite Based Augmentation Systems (SBAS) or Ground Based Augmentation System (GBAS). Moreover, the position can be computed using a small handheld receiver which can be taken everywhere. This technology has revolutionized our way of navigating. The impact on aviation is huge: planes are now able to determine their position all around the world with very high accuracy. Furthermore, in order to ensure that the computed position does not have an error above a certain threshold, integrity concepts have been developed (e.g. Receiver Autonomous Integrity Monitoring (Walter, Enge 1995), which aims to detect faulty satellites).

Commercial air traffic is assumed to multiply twice in the next ten years within European airspace. Airplanes are flying on sky highways, controlled by Air Traffic Control (ATC), telling them if they can or cannot move to another altitude or another airway. For the moment, in order to prevent collision, ATC requires minimum distances between planes. In the enroute phase, these minimums are usually $5 \mathrm{~nm}$ laterally and $1000 \mathrm{ft}$ vertically under RSVM Minima (FAA Order 7110.65). This makes the sky safe but limits the number of civil aircraft on a given airway. Here, precise knowledge of the distance between aircraft would help to decrease the interval between them and allow more planes to fly in the sky simultaneously. Autonomous and safe formation flying of two or more aircraft is thus a very promising technology for many future systems that need precise and safe baseline measurements. A system which would provide such a precise and robust distance vector could help to decrease separation and thus increase the traffic flow during en-route and approach phases.

From a military point of view, such a system could also be very effective for the interception of a civil plane by a fighter. When a civil plane does not respond to controllers, and may be a potential danger (e.g. flying over restricted area), a fighter automatically takes off and reaches the uncooperative plane. For the time being, the fighter detects the plane in instrument weather conditions by using a primary radar. It sends a high frequency radio sweep that is reflected back to the radar aerial by the civil aircraft. The fighter can then position its target and fly towards it. The fact that the plane does not need to be cooperative is the main advantage of this method. The drawbacks, however, are the weight of the radar and its expensive price and maintenance cost.

The first goal of GPS was to provide absolute position all around the world. It can, however, also be used to compute the relative position between two objects. If the fighter can compute the baseline between itself and the civil aircraft, it could then intercept it without visibility. Nevertheless, this data must be precise and reliable, which means that navigation integrity is a critical parameter to ensure safety. To compute the baseline between two objects, a method called Double Difference (Goad 1996) was developed using measurements between two receivers and two satellites. Thus, in the case of a master and a rover flying together, the master just has to send its GPS measurements to the rover which in turn computes the baseline between them using the double difference technique. The major advantage of this method lies in removing most of the errors inherent to GNSS signals and thus allowing high accuracy in the computed baseline. However, having the baseline is not enough; we also need a measure of the quality of said estimate. Thus, to ensure integrity, protection levels (PL) for the distance vector which have to be compared with maximum allowable alarm limits will be required.

Within the framework of the implementation of datalinks, new ways of digital communications between aircraft are being developed as part of the European NEWSKY project, for example LDACS (Schnell et al. 2010). It aims is to create datalinks and networks in the sky, where planes will be able to exchange data between them and with the ground using digital communications. In this context, pseudoranges could be easily added to the messages sent, without the need of specific equipment. The double difference method would then be a powerful tool for pilots to know reliably where planes are located around them.

\section{Code based double differences}

One of the standard methods to determine the baseline between two GNSS receivers is the formation of double differences (DD) to remove correlated errors (Cosentino et al. 2006). Since positioning based on carrier phase measurements is not well accepted in the aviation community, we form the double differences based on code measurements. For this, we assume that the pseudorange between receiver $k$ and satellite $p$ can be modeled as:

$$
\rho_{k}^{p}(t)=r_{k}^{p}+c\left(\delta^{p}(t)-\delta_{k}(t)\right)+Q_{k}+\gamma_{\text {iono }}(t)+\gamma_{\text {tropo }}(t),
$$

where $r_{k}^{p}$ being the geometric range; $\delta^{p}$ and $\delta_{k}$ - the satellite and receiver clock biases; $Q_{k}$ - the receiver noise; $\gamma_{\text {iono }}$ - the error induced by the ionosphere and $\gamma_{\text {tropo }}$ - the error induced by propagation through the toposphere. In order to suppress errors correlated with one particular satellite such as the clock bias $\delta^{p}$, we form the Single Difference (SD) pseudorange, between the measurements of two receivers $k$ and $m$. Because of the proximity of our two receivers, we assume for the remainder of this section that the ionospheric and 
tropospheric delays between receiver $k$ and $m$ are the same and can also be removed:

$$
S D_{k m}^{p}=\rho_{m}^{p}-\rho_{k}^{p}=r_{k m}^{p}+Q_{k m}+c\left(\delta_{k}-\delta_{m}\right)
$$

is the difference between raw pseudoranges of receivers $k$ and $m$, for satellite $p$. It corresponds to the difference of geometric ranges, plus a term $Q_{k m}$ of noise and multipath, and the remaining receiver clock biases. The satellite bias $\delta^{p}$ is common to the two pseudoranges and was thus canceled by the difference. We can also express the SD as a function of the baseline between the two receivers by linking the baseline with differential geometric ranges $r_{\mathrm{km}}^{p}$. This is analogue to the principles of interferometry.

Figure 1 depicts a single GPS-interferometer interacting with a single satellite. An interferometer takes into account the interferences between two coherent waves; in our case, we assume that the signal reaching receiver $k$ is equal to the signal reaching receiver $m$ plus an additional signal corresponding to the differential geometric ranges $r_{k m}^{p}$. Let $k$ and $m$ be the phase center of the antennas, and $\vec{b}$ the unknown baseline vector between them. Knowing that satellite $p$ is at a distance of about $20000 \mathrm{~km}$, we can assume that the paths of propagation

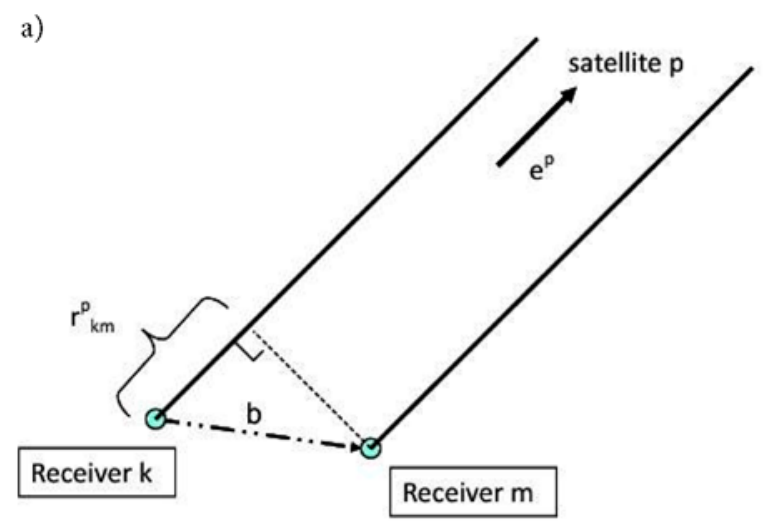

b)

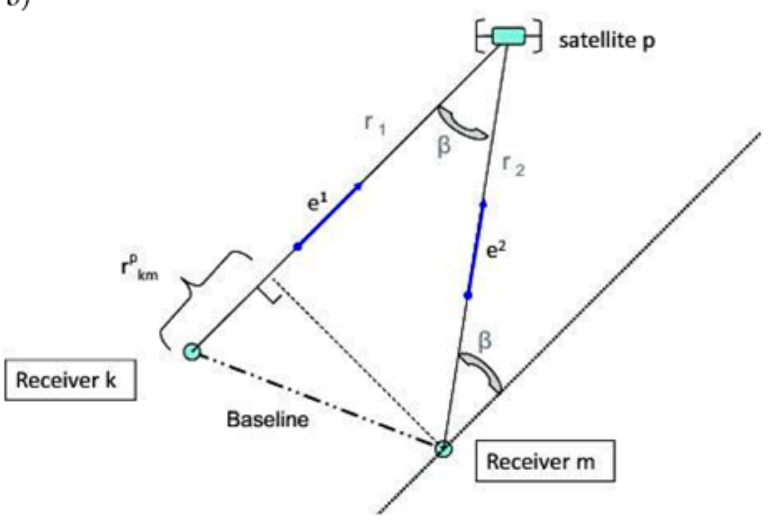

Fig. 1. a) - GPS interferometer with one satellite. By assuming that the two receivers are looking to the satellite $p$ with the same angle, we can simply express the baseline as a function of the angle and the differential geometric ranges $\left.r_{k m}^{p} ; b\right)$ - GPS interferometer's bias with one satellite between the satellite and the two antennas are quasi parallel. We also know the line of sight vector $e^{p}$ to satellite $p$. Figure 1(a) shows us that the Single Difference can easily be expressed as the projection of the relative position vector onto the line of sight vector to $\backslash$ satellite $p$.

The differential geometric ranges $r_{k m}^{p}$ can thus be expressed as the scalar product between unit vector $e^{p}$ and baseline $\vec{b}$ :

$$
r_{k m}^{p}=\vec{e} \vec{p} \cdot \vec{b}
$$

This means for the single difference equation:

$$
S D_{k m}^{p}=\vec{e} \vec{p} \cdot \vec{b}+Q_{k m}+c\left(\delta_{k}-\delta_{m}\right)
$$

We still have the error due to the receiver's clock bias in this equation. To remove this term, the difference between the SD of the same two receivers with another satellite $q$ is formed:

$$
S D_{k m}^{q}=\overrightarrow{e^{q}} \cdot \vec{b}+Q_{k m}+c\left(\delta_{k}-\delta_{m}\right)
$$

then differentiate the two SD Equations yielding a double difference:

$$
\begin{aligned}
& D D_{k m}^{p q}=S D_{k m}^{p}-S D_{k m}^{q}=\left(\rho_{m}^{p}-\rho_{k}^{p}\right)-\left(\rho_{m}^{q}-\rho_{k}^{q}\right)= \\
& \overrightarrow{\vec{p}} \overrightarrow{\vec{q}}) \cdot \vec{b}+Q_{k m}^{p q},
\end{aligned}
$$

with $Q_{k m}^{p q}=\left(Q_{k}^{p}-Q_{m}^{p}\right)-\left(Q_{k}^{q}-Q_{m}^{q}\right)$ corresponding to the noise and the multipaths of all the receivers. Dropping the vectorial notations and ignoring the noise term, the double difference becomes:

$$
D D_{k m}^{p q}=(\vec{e} p-\vec{e}) \cdot \vec{b}
$$

which can be solved using standard least squares methods. The use of the matrix notation yields the following equation:

$$
D D_{p r}=H \cdot \vec{b}
$$

Note that the geometry matrix $H$ contains the difference between unit vectors to different satellites for all possible satellite combinations. Therefore, this method does not have the inherent shortcomings of the GNSS position solution. In the GNSS position solution, due to the unavailability of measurements from below the user, the vertical precision of the solution is less than the horizontal. Here due to the differencing this vertical dilution of precision is mitigated. Baseline $b$ can then be obtained by using $S$, the pseudo-inverse of geometry matrix $H$ :

$$
\begin{aligned}
& b=S \cdot D D_{p r}, \\
& S=\left(H^{T} H\right)^{-1} H^{T} .
\end{aligned}
$$


Beyond a certain baseline the assumption that the paths of propagation between the satellite and the two antennas are parallel is no longer valid. We analyzed the impact of this hypothesis, by taking into account the angle $\beta$ between the two paths of propagation (Fig. 1b) to compute the remaining error brought by this hypothesis, and thus correct our estimated baseline. The baseline is calculated again using the double difference method, but taking into account angle $\beta$ between the two paths of propagation. Baseline $\vec{b}$ can be expressed as

$$
\vec{b}=r_{1} \cdot \vec{e}-r_{2} \cdot \vec{e}
$$

The scalar product between baseline $\vec{b}$ and unit vector $e_{1}$ becomes:

$$
\vec{b} \cdot \vec{e}=\left(r_{1} \cdot \vec{e}-r_{2} \cdot \vec{e}\right) \cdot \vec{e}=r_{1}-r_{2} \cdot \vec{e} \cdot \overrightarrow{\overrightarrow{2}} \cdot \vec{e},
$$

knowing that $\vec{e}$ and $\vec{e}$ are unit vectors:

$$
\begin{aligned}
& \overrightarrow{\overrightarrow{2}} \cdot \overrightarrow{\vec{e}}=\cos \left(\beta^{p}\right) ; \\
& \vec{b} \cdot \vec{e}=r_{1}-r_{2}+\underbrace{r_{2}\left(1-\cos \beta^{p}\right)}_{\text {additional-term }} .
\end{aligned}
$$

Thus, a remedial term to the former single difference $S D_{k m}^{p}$ must be added, which becomes:

$$
S D_{k m}^{p}+r_{m}^{p}\left(1-\cos \left(\beta^{p}\right)\right)=\vec{b} \cdot \vec{e} \vec{p}
$$

The double difference then becomes:

$$
D D_{k m}^{p q}+r_{m}^{p}\left(1-\cos \beta^{p}\right)-r_{m}^{q}\left(1-\cos \beta^{q}\right)=\left(e^{p}-e^{q}\right) \cdot \vec{b} .
$$

To measure the impact of this error, we drew the baseline correction (computed using equation 15), as a function of the distance for the constellation of midnight March the 1st, 2011 as observed at Oberpfaffenhofen, Germany. The result is depicted in Figure 2. It is evident that the hypothesis of parallel propagation paths created at the beginning has a large impact for long baselines.

\section{Error considerations and protection of the baseline}

In Section 2 we assumed that the ionospheric and tropospheric residuals were eliminated, and that the noise and multipath were negligible. We now consider the double difference method without ignoring the noise and multipath and by taking into account the ionosphere and troposphere residuals. We can thus reformulate equation 6 as:

$$
D D_{k m}^{p q}=\left(e^{p}-e^{q}\right) b+Q_{k m}^{p q}+\delta_{\text {iono }}^{p q, k m}+\delta_{\text {tropo }}^{p q, k m},
$$

where:

$$
Q_{k m}^{p q}=\left(Q_{k}^{p}-Q_{m}^{p}\right)-\left(Q_{k}^{q}-Q_{m}^{q}\right)
$$

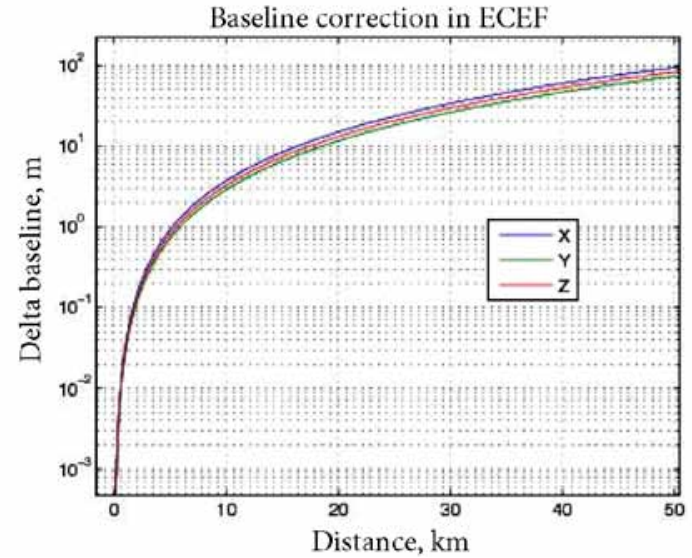

Fig. 2. Illustration of the impact of the first hypothesis (saying that the paths of propagation between the satellite and the two antennas are parallel. The Y semi-log axis represents the error introduced in the baseline because of this hypothesis. The scenario at stake here is: a rover gets away equally in each direction (X, Y, Z) from the master. We can see that this hypothesis is true up to a certain distance, depending on the wanted precision

for the total noise and multipath.

$$
\delta_{k m}^{p q}=\left(\delta_{k}^{p}-\delta_{m}^{p}\right)-\left(\delta_{k}^{q}-\delta_{m}^{q}\right)
$$

for the ionospheric and the tropospheric residuals.

\subsection{Receiver noise and multipath}

The error behaviour of a single difference code measurement is very similar to the behavior experience when using differential GNSS. The uncorrelated errors of two measurements propagate as the addition of two Gaussian random variables, i.e. their variances and mean are additive. As such, the noise and multipath models used in GBAS are applicable for considering a single difference as well. For the double difference error model, the results from the two single difference error considerations need to be propagated again.

As in GBAS, we treat the multipath and noise error as having a common Gaussian distribution with zero mean and an elevation dependent variance. The models were derived by G. A. McGraw et al. (2000) and can also be found in the airborne standards for GBAS, RTCA DO-253C:

$$
\sigma_{\in, j}^{2}=0.15+0.43 e^{\frac{\theta_{j}}{6.9^{\circ}}},
$$

where $\theta_{j}$ is the elevation angle (in degrees) for satellite $j$. This sigma represents the airborne contribution of the multipath and noise for one receiver-satelite pair. It also includes the effects of a Hatch filter (Hatch 1982) with a 100s smoothing time constant.

\subsection{Ionosphere residuals}

The ionosphere layer is a dispersive medium composed of ionized gas located in the upper atmosphere. Due to 
the sun's radiation, free electrons and ions are generated and cause phase advance and group delay to electromagnetic waves, like GPS signals. This layer is not uniform and varies in time and in space. To quantify the spatial decorrelation of the ionosphere, a vertical ionospheric gradient was introduced by M. Luo et al. (2002). The value of this gradient, expressed in $\mathrm{mm} / \mathrm{km}$, corresponds to the difference of ionospheric delays on the L1 frequency between two points at a distance of one kilometer. Thus, by doing a single difference between two receivers, the ionospheric delay is not totally removed, in the presence of gradients ionospheric residuals may remain.

Figure 2 displays the impact of the double difference on the ionosphere. We see that the two single differences bring each one a ionospheric residual. In order to be conservative, we assume that there is no correlation between the two ionospheric residuals.

The nominal ionospheric gradient is assumed to be a random variable (Christie et al. 1999) the distribution of which is Gaussian. We assume that the Single Difference operation carried out in our method has nearly the same impact on the ionospheric and tropospheric delay as the GBAS operation. The residual ionospheric uncertainty for one satellite is then expressed in equation 18 (RTCA DO-253C: 2008); it depends on the standard deviation of the nominal vertical ionospheric gradient $\sigma_{v e r t-i o n o-g r a d}$, the satellite elevation angle through the obliquity factor FPP and distance $\chi_{\text {air }}$ between the two flying objects. If carrier phase smoothing is used, we also have to add a term representing the code-carrier divergence due to the ionospheric divergence that occurs when the planes traverse a ionosphere gradient over one smoothing time constant $\tau$.

$$
\sigma_{\text {iono }, S D}^{2}=\left[F P P \sigma_{\text {vert-iono-grad }}\left(\chi_{\text {air }}+2 \tau v_{\text {air }}\right)\right]^{2} \text {. }
$$

The obliquity factor, sometimes also referred to as an elevation mapping function, accounts for an increased path length through the ionosphere at low elevations. $\sigma_{\text {vert-iono-grad }}$ represents the gradient of the normally distributed ionosphere $[\mathrm{mm} / \mathrm{km}]$; its value is set as $5 \mathrm{~mm} / \mathrm{km}$, which is, according to M. Luo et al. (2002), a conservative one sigma value for vertical ionosphere spatial decorrelation. As depicted in Figure 2, ionospheric residuals depend on $\chi_{\text {air }}$ the slant range distance between the two aircraft. We are, however, using two satellites for the double difference instead of one; thus another $\sigma_{\text {res,iono }}$ has to be taken into account. Assuming the two gradients are uncorrelated, the following is obtained:

$$
\begin{aligned}
& {\left[\sigma_{k m}^{p q}\right]_{\text {iono }, D D}^{2}=\left[F P P_{k m}^{p} \sigma_{\text {vert-iono-grad }}\left(\chi_{\text {air }}+2 \tau v_{\text {air }}\right)\right]^{2}+} \\
& {\left[F P P_{k m}^{q} \sigma_{\text {vert-iono-grad }}\left(\chi_{\text {air }}+2 \tau v_{\text {air }}\right)\right]^{2}}
\end{aligned}
$$

\subsection{Troposphere residuals}

The troposphere is the lowest layer of the atmosphere and contains nearly all the moisture present within the atmosphere. More importantly, it is a non-dispersive medium which also delays electromagnetic signals. Tropospheric delay consists of two components: a dry component, which is only dependent on dry constitutent gases of the atmosphere and accounts for approximately $90 \%$ of the delay, and a wet component, which depends only on the moisture content of the atmosphere and accounts for the remaining effect of the delay (Leva et al. 2006).

Similarly to the ionosphere, some tropospheric residuals remain after taking the single difference between two receivers due to local variations of the troposphere's composition. These errors can also be modeled as a Gaussian random variable. On the contrary to the ionosphere, the tropospheric delay residual error depends on the height difference between the receivers. Residual tropospheric uncertainty can be expressed as (as defined in DO-253C):

$\sigma_{\text {tropo }, S D}^{2}=\left[\sigma_{n} h_{0} \frac{10^{-6}}{\sqrt{0.002+\sin \theta_{j}^{2}}}\left(1-e^{\left(-\frac{\Delta h}{h_{0}}\right)}\right]^{2}\right.$.

This variance depends on a refractivity uncertainty $\sigma_{n}\left[m^{-1}\right]$, a tropospheric scale height $h_{0}\left[\mathrm{~m}^{-1}\right]$, the elevation of the satellite $e l[\mathrm{rad}]$, and the difference in altitude between airborne $\Delta h[m]$. For the refractivity uncertainty and the tropospheric scale height, we used $\sigma_{n}=33$ and $h_{0}=15730 m$ from T. A. Skidmore and F. van Graas (2004). As for the ionospheric uncertainty, we are using two satellites for the double difference instead of one; we then add the two variances:

$$
\begin{aligned}
& {\left[\sigma_{k m}^{p q}\right]_{\text {tropo }, D D}^{2}=\left[\sigma_{n} h_{0} \frac{10^{-6}}{\sqrt{0.002+\sin \left(\theta_{k m}^{p}\right)^{2}}}\left(1-e^{\left(-\frac{\Delta h}{h_{0}}\right)}\right)\right]^{2}+} \\
& {\left[\sigma_{n} h_{0} \frac{10^{-6}}{\sqrt{0.002+\sin \left(\theta_{k m}^{q}\right)^{2}}}\left(1-e^{\left(-\frac{\Delta h}{h_{0}}\right)}\right]\right.}
\end{aligned}
$$

\subsection{Protection levels}

To simplify the notation, we drop the $p, q$ and $m, k$ indices that were used previously. We assume that the errors described in the previous paragraphs are independent and uncorrelated. Therefore, the error of the double differences can be expressed as

$$
\text { error } D D=Q+\delta_{\text {tropo, } D D}+\delta_{\text {iono, } D D} .
$$

The variance of this error is:

$$
\operatorname{var}(\text { error } D D)=\operatorname{var}\left(Q+\delta_{\text {iono, } D D}+\delta_{\text {tropo }, D D}\right) \text {. }
$$


We have seen that $Q$ is a sum of four uncorrelated random variables with a Gaussian distribution, and we can thus directly define its variance:

$$
\operatorname{var}(Q)=4 \sigma_{\epsilon}^{2},
$$

where $\sigma_{\in}^{2}$ is the variance of the noise and multipath error for one receiver. We assume that the variance is the same for both receivers.

Using the previously defined variances of the ionospheric and tropospheric residuals we can obtain the variance of the total range error:

$$
\sigma_{\text {error } D D}^{2}=4 \sigma_{\epsilon}^{2}+\sigma_{\text {iono, } D D}^{2}+\sigma_{\text {tropo }, D D}^{2}
$$

With the errors regarded as randomly distributed Gaussian variables, now only the impact of the constellation geometry on our variance has to be taken into account; therefore, the errors into the position domain through the pseudo-inverse $S$ of the geometry matrix $H$ (equation 10) have to be mapped. At the end, we multiply the result by factor $\mathrm{K}$, representing the desired integrity risk ( $N$ here represents the number of satellites in view):

$$
P L_{i}=K \sqrt{\sum_{k=1}^{N-1} S_{i, k}^{2}\left(4 \sigma_{\in}^{2}+\sigma_{\text {iono, res } D D}^{2}+\sigma_{\text {tropo, res } D D}^{2}\right)} .
$$

Note that, in comparison with GBAS, the protection levels will be larger since the double difference consists of four individual measurements. We have now obtained protection levels for the three dimensions, corresponding to a certain integrity risk dependent on the value of $K$. These protection levels (PL) depend only on the noise, the tropospheric and ionospheric residuals, and the geometry of the constellation. In all our simulations, we use the factor $K=5.32$, which corresponds to an integrity risk of $10^{-7}$ for each of the three components, yielding a total risk of $3 \times 10^{-7}$. The derived protection levels are given an acceptable risk for an approaching aircraft. To be meaningful, the coordinate system, in which the PLs are computed is centered at the user and the baseline. An illustration of the concept is shown in Figure 3.

\section{Static sites}

In order to validate the application of the double difference method, we used the data from two GBAS reference receivers at Braunschweig (Dautermann et al. 2011). We used the measurements from BR01 and BR02, collected during 10 hours of the 17th of December, 2009 (from midnight to 10 a.m.). Figure 4 displays the results of our computation. It gives the error of the euclidean distance (for the three dimensions) over a period of 10 hours.

It is illustrated in Figures 4 (b) and 4 (c) that the error is $\pm 1.5 \mathrm{~m}$. According to the distance of 775.49 meters between the 2 stations, we can say that the ionospheric and tropospheric residuals after the double difference are

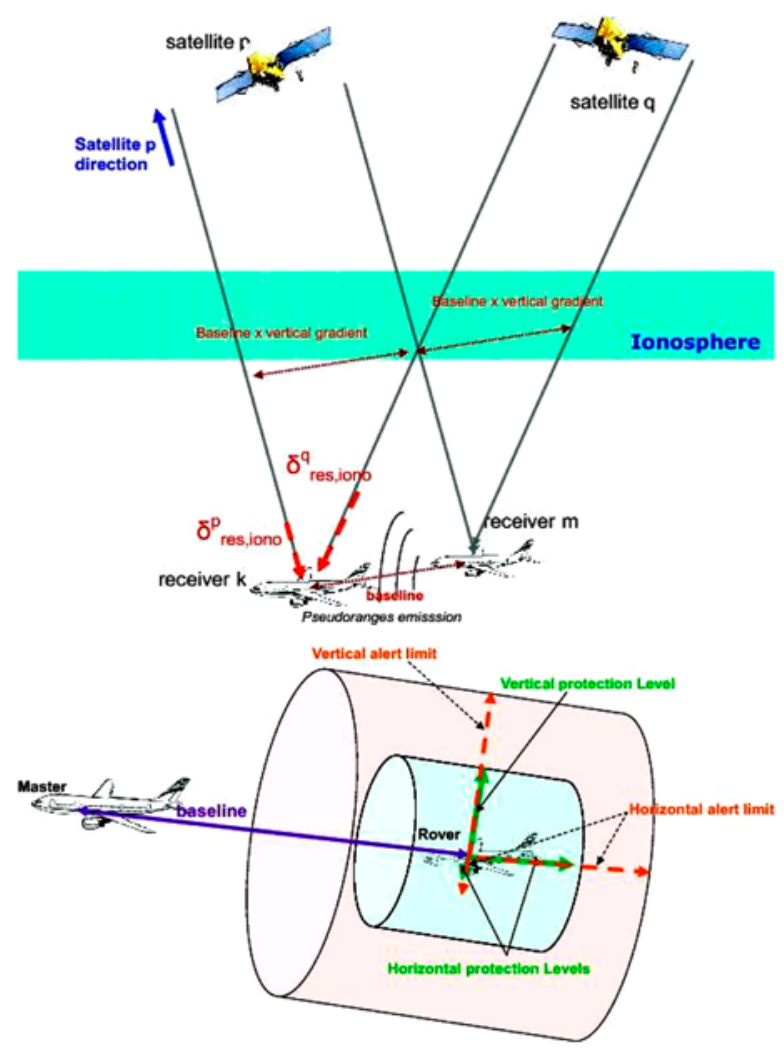

Fig. 3. Protection levels in relative navigation. We can see it as an area where the rover is located relatively to the master

very small. Indeed, at this distance, the ionosphere and troposphere between these two points are strongly correlated. Among the possible errors the noise of the two receivers remains. We can also assume that some ground multipath also have an impact on the error (the receivers are close to the ground). Moreover, the fact that the two objects are static increases the impact of the multipath. These assumptions are validated by Figure 4(a) representing the horizontal error after smoothing of the pseudoranges with a time constant of $30 \mathrm{sec}$. We can indeed notice that the error after smoothing is almost divided by two.

Figure 4(b) depicts the error in North-East-Up side. It is interesting to notice that, as opposed to the error for a standalone positioning, the Up error is not much higher than the other ones. For the standalone position solver, the vertical error component was much higher than the horizontal ones because of the constellation geometry. This is not the case for the double difference method; the geometry used in the least squares solution is a differential geometry which mitigates the effect. Figure 4(c) illustrates that the protection levels are always well above the errors and very conservative. The protection levels react in the same way as the error, increasing when the error increases. Since the two sites are static, the main parameter for the computation of the protection levels is the number of satellites. 
a)

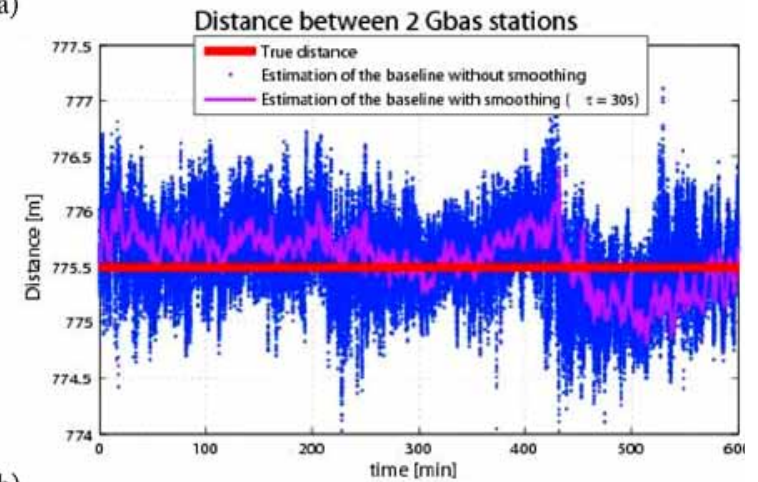

b)

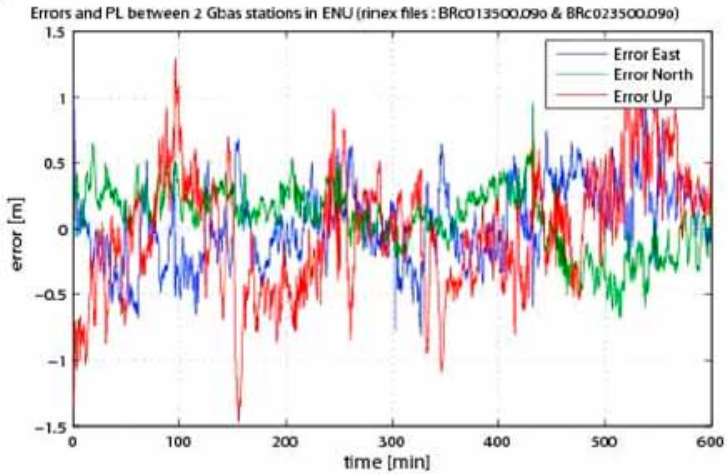

c)

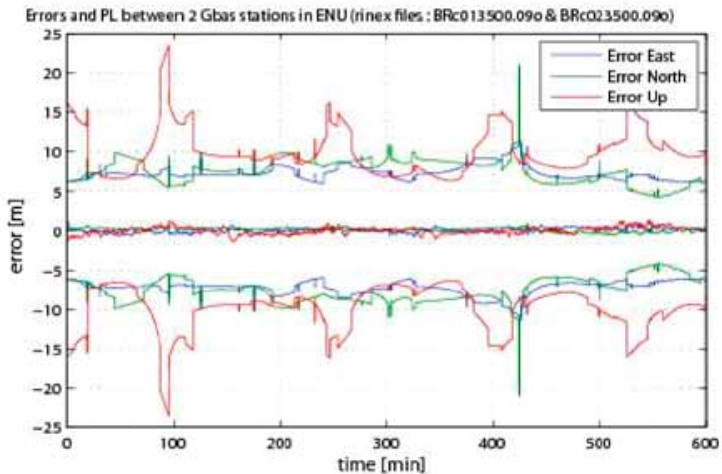

Fig. 4. a) - distance error between two GBAS stations before and after smoothing $(\tau=30 \mathrm{sec})$. We can notice that the error is nearly divided by two; b) - errors in ENU between two GBAS stations with smoothing of the pseudoranges $(\tau=30 \mathrm{sec})$. The three components all have the same order of magnitude; c) - errors and protection levels between 2 GBAS stations. The error is smoothed $(\tau=30 \mathrm{sec})$; we can notice that the spike $(t=$ $120 \mathrm{~min}$ ) correspond to less satellites

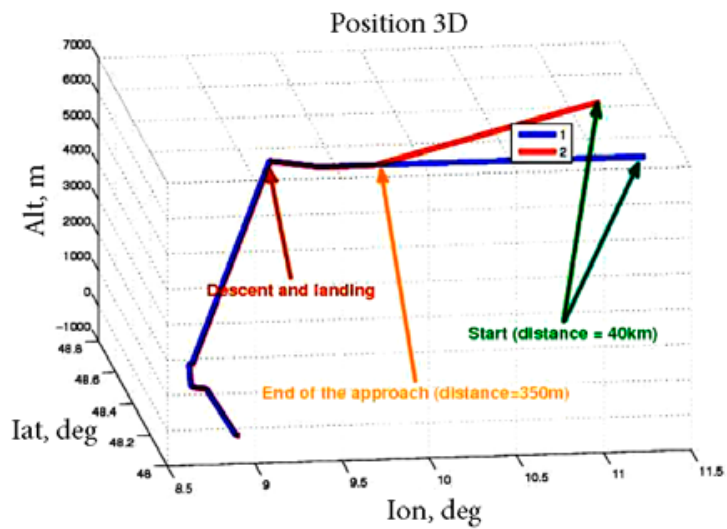

Fig. 5. Presentation of the simulated trajectory; the rover gets closer to the master (up to $350 \mathrm{~m}$ ) and then flies alongside. They at the end land together

\section{Intercept mission}

One of the special cases of interest is the interception scenario. Here, a military fighter jet intercepts a civilian airplane and escorts it to the landing site. We used a dual Spirent GSS7790 constellation simulator to simulate the same constellation for both the intercepting aircraft (hereinafter referred to as rover) and the aircraft to be intercepted (hereinafter referred to as master). The rover is the flying object which wants to define the baseline to approach and intercept the master. The rover gets closer to the master (up to $350 \mathrm{~m}$ ), then flies alongside escorting the master to a landing. At the end they land together. Their trajectories are shown in Figure 5. During the simulations we used the GPS L1 signal, which is appropriate for aviation use. We arbitrarily decided to use the GPS constellation of the first of March, 2011. The simulations began at $22 \mathrm{~h} 30 \mathrm{~min}$ and lasted for about 30 minutes.

Figure 6 depicts the errors in ENU and the protection levels in the case where the only error is receiver noise. The protection levels react to the loss of satellites and to the distance between the two vehicles. Indeed, after the approximation phase, we can see that the protection levels stay the same since the constellation does not change and the distance between the rover and the master remains constant. Also, as expected, the only error remaining is the receiver noise, equal to a white noise.

As explained in section 3.1, during the en-route phase, only fuselage multipath impacts the pseudorange error. Thus, the aim of the scenario is to depict the effect of such a multipath on the baseline computation.

The first step in our scenario is to define the fuselage multipath on the approach trajectory. We did not want to use the input model as described in the previous section, but rather wanted to see if a bias is still protected by our approach. To do so, the approach from A. Hornbostel et al. (2010) and A. Steingass et al. (2004) for fuselage

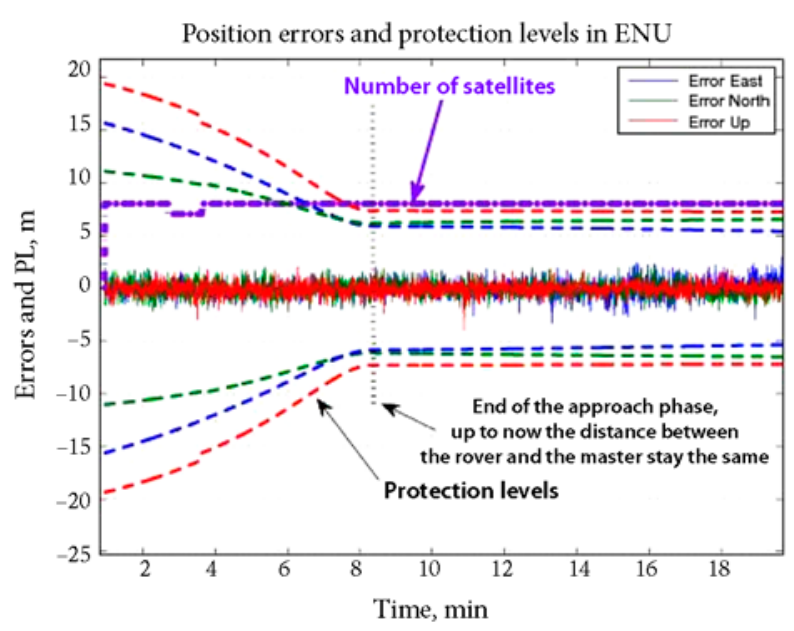

Fig. 6. Errors and protection levels in ENU for the simulated trajectory without smoothing. The number of visible satellites has been added 
multipath analysis was used. The authors show that the dominant component of fuselage multipath corresponds to a fixed offset of $1.5 \mathrm{~ns}(0.45 \mathrm{~m})$ and an attenuation of $14.2 \mathrm{~dB}$ with a random phase. Since the DLR's spirent is composed of two units, we are able to simulate 24 channels on the L1 band. We have thus added, for each visible satellite (we take the worst case and assume that all the signals are affected by a Multipath), a corresponding multipath with the specifications defined above (we simulate two signals for each satellite: the light of sight and the multipath). In our scenario, the multipath appears three minutes after the start of approach and is then canceled after 15 minutes.

We can already maintain that the error introduced by fuselage multipath is not going to be huge. Indeed, the fuselage multipath we have implemented is modeled as a fixed offset of $0.45 \mathrm{~m}$ with an attenuation of $14.2 \mathrm{~dB}$ and a random phase. This multipath is furthermore added on each satellite's signal. Due to the double difference, the total added bias can reach a maximum of $4 \times 0.45 \mathrm{~m}$ on a receiver. The phase of the multipath is Gaussian, so if during the single difference the multipath received by the two planes are in phase, they will cancel themselves out. However, if their phases are opposite, multipath will add up and create a $0.90 \mathrm{~m}$ range bias. After the double difference the added bias could then be $1.80 \mathrm{~m}$ at most for the model we plan to use.

Figure 7 depicts the impact of the multipath that we have implemented. First, we can notice that, as expected, protection levels are not influenced by the multipath. Also, as expected, the baseline has no influence on the errors. Secondly, fuselage multipath do not add a much higher than a one meter error on the baseline. Figure 7(b) illustrates the distance error between the two planes (the error is computed as the euclidean distance of the three dimensional errors). We can see that the error added on the distance is within 1.2-1.6 meters, in our worst-case scenario.

\section{Conclusions}

Firstly, the double difference method in the case of a single constellation (GPS), and a single frequency (L1) and its application to aviation were analysed. We chose to use only the L1 frequency because for the moment it is the only one available within the aeronautical navigation band. We also chose to use the code measurements instead of the carrier phase; indeed, even if the code is less precise than the phase, it is more robust and thus more suitable to the derivation of protection levels. For this reason, it is more accepted in civil aviation. We have seen that for one vehicle to compute the baseline to another vehicle, it needs to send to it its GNSS observables. For this one of the future datalink concepts like LDACS can be used. a)

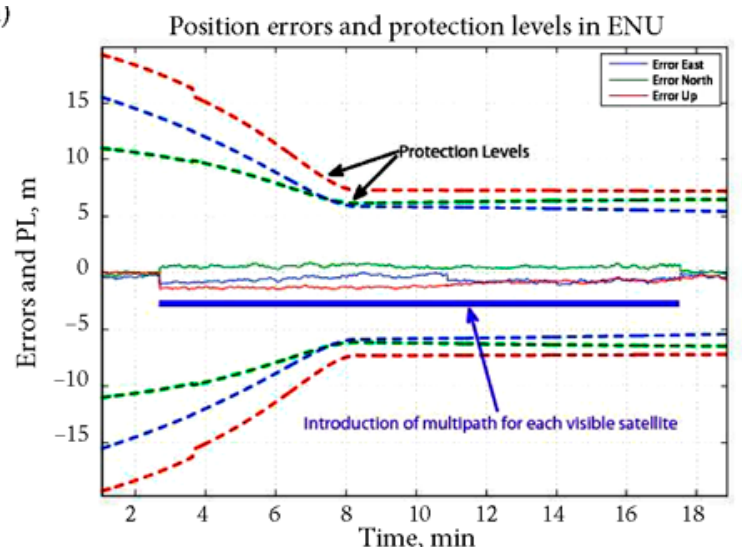

b)

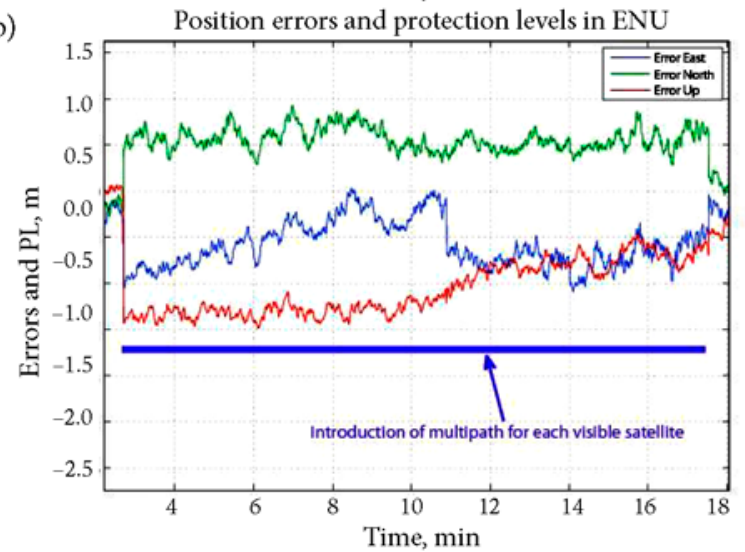

Fig. 7. a) - depicts the impact of multipath during the approach phase. The pseudoranges are unsmoothed; b) - is a zoom of Figure (a). We can see that the added error is below two meters

The double difference method is very suitable to such an application since differencing removes all errors that are common to the receiver and satellite. However, care must be taken if errors that are not common to both receivers occur. For example, a correlator malfunction in one of the receivers could lead to a deformation of the autocorrelation peak and a multipath like bias on one recever-satellite link. This event can be covered by a receiver fault hypothesis protection level, similar to the $H 1$ hypothesis in GBAS. For the exact determination of such a bias, detailed investigations of receiver malfunctions and their causes are required, which would exceed the scope of the investigation presented here.

We have shown that the protected baseline method using the double difference could be suitable for safe baseline estimation between datalinked vehicles. Especially, when combined with concepts like Advanced Receiver Autonomous Integrity Monitoring (Rippl et al. 2011), it could contribute to a reduction of separation between aircraft in enroute and approach phases without requiring an additional costly infrastructure.

\section{Acknowledgements}

This work was performed as part of a Master's thesis at the German Aerospace Center, Institute of Communications 
and Navigation, Oberpfaffenhofen, 82234 Wessling, Germany, in collaboration with Ecole Nationale de l'Aviation Civile (ENAC) in fullfillment of the requirements for a Master's degree. The authors would like to thank Christophe Macabaiau an Anais Martineau for supervising this work from ENAC's side and to E. Schielin of the German Aerospace Center, Department of Navigation for funding.

\section{References}

Christie, J. R. I.; Ko, P. Y.; Hansen, A., et al. 1999. The effects of local ionospheric decorrelation on LAAS: theory and experimental results, in ION NTM, Proceedings of the 1999 National Technical Meeting of The Institute of Navigation, January 1999, San Diego, CA, 769-777.

Cosentino, R. J.; Diggle, D. W.; De Haag, M. U., et al. 2006. Understanding GPS: Principles and Applications. Artech House, 379-458.

Dautermann, T.; Felux, M.; Grosch, A. 2011. Approach service type D evaluation of the DLR GBAS testbed, GPS Solutions 1-13. http://dx.doi.org/10.1007/s10291-011-0239-3

Goad, C. 1996. Short Distance GPS Models, in A. Kleusberg, P. Teunissen (Eds.). GPS for Geodesy. Lecture Notes in Earth Sciences, vol. 60. Springer, 239-262. http://dx.doi.org/10.1007/BFb0117684

Hatch, R. 1982. The synergism of GPS code and carrier measurements, in Proceedings of the Third International Geodetic Symposium on Satellite Doppler Positioning, 8-12 February 1982, New Mexico State University 2: 1213-1232.

Hornbostel, A.; Steingass, A.; Crisci, M., et al. 2010. GNSS receiver performance assessment with a realistic aeronautical channel model, in Satellite Navigation Technologies and European Workshop on GNSS Signals and Signal Processing (NAVITEC), 2010 5th ESA Workshop, 8-10 December 2010, Noordwijk. http://dx.doi.org/10.1109/NAVITEC.2010.5708047

Leva, J.; De Haag, M. U.; Van Dyke, K. 2006. Understanding GPS: Principles and Applications. Artech House, 301-375.

Luo, M.; Pullen, S.; Akos, D., et al. 2002. Assessment of ionospheric impact on LAAS using WAAS Supertruth data, in ION AM, Proceedings of the 58th Annual Meeting of The Institute of Navigation and CIGTF 21st Guidance Test Symposium, 24-26 June 2002, Albuquerque, NM.
McGraw, G. A.; Murphy, T.; Brenner, M., et al. 2000. Development of the LAAS accuracy models, in ION GPS, Proceedings of the 13th International Technical Meeting of the Satellite Division of The Institute of Navigation, 19-22 September 2000, Salt Lake City, UT.

Rippl, M.; Spletter, A.; Günther, C. 2011. Parametric performance study of advanced receiver autonomous integrity monitoring (ARAIM) for combined GNSS constellations, in Proceedings of the 2011 International Technical Meeting of the Institute of Navigation (ITM2011), 24-26 January 2011, San Diego, CA.

RTCA DO-253C: 2008. Minimum Operational Performance Standards for GPS Local Area Augmentation System Airborne Equipment. Tech. Rep. 253C: Radio Technical Commission for Aeronautics.

Schnell, M.; Franzen, N.; Gligorevic, S. 2010. L-dacs1 laboratory demonstrator development and compatibility measurement set-up, in Digital Avionics Systems Conference, 3-7 October 2010, Salt Lake City, UT.

Skidmore, T. A.; Van Graas, F. 2004. An investigation of tropospheric errors on differential GNSS accuracy and integrity, in ION GNSS, Proceedings of the 17th International Technical Meeting of the Satellite Division of The Institute of Navigation, 21-24 September 2004, Long Beach, CA, 2752 - 2760.

Steingass, A.; Lehner, A.; Perez-Fontan, F., et al. 2004. A high resolution model for the aeronautical multipath navigation channel, in Position Location and Navigation Symposium, PLANS 2004, 26-29 April 2004, 357-365. http://dx.doi.org/10.1109/PLANS.2004.1309017

Walter, T.; Enge, P. 1995. Weighted RAIM for Precision Approach, in Proceedings of the 8th International Technical Meeting of the Satellite Division of the Institute of Navigation (ION GPS 1995), 12-15 September 1995, Palm Springs, CA. 